\title{
Trajectory Planning of Ammunition Loading Robot Based on Cubic-B Spline
}

\author{
Yang LI ${ }^{1, a, ~ *, ~ D a ~ X U ~}{ }^{2, b}$, Ye LUO ${ }^{3, c}$ \\ ${ }^{1}$ Department of Arms Engineering, Academy of Armored Force Engineering, Beijing, 100072, China \\ ${ }^{2}$ Department of Arms Engineering, Academy of Armored Force Engineering, Beijing, 100072, China \\ ${ }^{3}$ Department of Arms Engineering, Academy of Armored Force Engineering, Beijing, 100072, China \\ a18510788818@163.com, bxyxd@sina.com, ${ }^{c} 1518391486 @ q q . c o m$ *corresponding author \\ corresponding author
}

Keywords: Ammunition Loading Robot, Trajectory Plan, Cubic-B Spline, Obstacle Avoiding

\begin{abstract}
To solve the problem of continuous and steady work and obstacles avoidance of ammunition loading robot, the trajectory plan method of ammunition loading robot based on cubic-B spline has been put forward. The paper analyzed the solution of cubic-B spline, finishing the obstacle avoiding trajectory plan of ammunition loading robot with key control points in joint space based on cubic-B spline, and the ammunition loading robot joints' angular displacement, angular velocity and angular acceleration is simulated by dynamic simulation software, the results showing that the method can quickly generate obstacle avoidance trajectory and dynamic characteristics of the trajectory is fine, guaranteeing the ammunition loading robot work smoothly with no impact and avoid obstacles effectively.
\end{abstract}

\section{Introduction}

With the increasing caliber of gun, ammunition weight reached the limits of artificial loading and active service autoloader exists problems such as low efficiency and poor reliability, the ammunition loading robot has become a domestic and international hot topic of automatic loading technology research[1][2]. Ammunition loading robot is PRR-3R type six degrees of freedom robot in which working space there are obstacles breechblock, seat ring and so on. In order to avoid these obstacles in the working process, the end effector need pass precisely all the key obstacle avoiding points smoothly with no impact[3][4], using the traditional polynomial interpolation method can't guarantee good characteristics of trajectory when it passing the key obstacle avoiding points[5][6]. The paper fulfil the obstacle trajectory plan of ammunition loading robot based on cubic-B spline, simulating the joints' dynamics by simulation software, verifying it is practical to proceed trajectory plan of ammunition loading robot with multiple obstacle avoidance points based on cubic-B spline.

\section{Cubic-B Spline}

The $i$ th segment of cubic-B spline curve of ammunition loading robot trajectory is

$$
\theta_{i}(t)=X_{0}(t) P_{i-1}+X_{1}(t) P_{i}+X_{2}(t) P_{i+1}+X_{3}(t) P_{i+2}
$$

In type (1), $t$ is time, $X_{i}(t)$ is the basic function of cubic-B spline, $P_{i}$ is the control point of the cubic-B spline curve.

Since the whole trajectory is continuous, the values of adjacent cubic-B spline curves are equal at the connecting point:

$$
\theta_{i}(1)=\theta_{i+1}(0)
$$

In addition, at the connection of two adjacent cubic-B spline curve, the derivatives and the second derivatives is equal, that means the velocity and acceleration of end effector is continuous, so we can get:

$$
\theta_{i}^{\prime}(1)=\theta_{i+1}{ }^{\prime}(0), \quad \theta_{i}^{\prime \prime}(1)=\theta_{i+1}^{\prime \prime}(0)
$$


Taking the derivative of type (2) combined with type (3), we can get:

$$
\begin{aligned}
& X_{0}^{\prime}(1)=X_{3}^{\prime}(0), \quad X_{0}^{\prime}(0)=X_{1}^{\prime}(1), \quad X_{2}^{\prime}(1)=X_{1}^{\prime}(0), \quad X_{3}^{\prime}(1)=X_{2}^{\prime}(0) \\
& X_{0}^{\prime \prime}(1)=X_{3}^{\prime \prime}(0)=0, \quad X_{0}^{\prime \prime}(0)=X_{1}^{\prime \prime}(1), \quad X_{2}^{\prime \prime}(1)=X_{1}^{\prime \prime}(0)=0, \quad X_{3}^{\prime \prime}(1)=X_{2}^{\prime \prime}(0)
\end{aligned}
$$

By the Cauchy standard of cubic-B spline basic function, we can get:

$$
X_{0}(t)+X_{1}(t)+X_{2}(t)+X_{3}(t)=1
$$

Combined type (2) to type (5), the basic functions of the cubic-B spline curves are obtained:

$$
\begin{aligned}
& X_{0}(t)=1 / 6\left(-t^{3}+3 t^{2}-3 t+1\right) \\
& X_{1}(t)=1 / 6\left(3 t^{3}-6 t^{2}+4\right) \\
& X_{2}(t)=1 / 6\left(-3 t^{3}+3 t^{2}+3 t+1\right)
\end{aligned}
$$

In trajectory planning, the control points of the cubic-B spline curves are needed, and the key obstacle avoiding points of the joint space trajectory of ammunition loading robot must be given before solving the control point, that is, the key obstacle avoiding points of $M_{i}$. From type (6) we can get:

$$
\theta_{i-1}(1)=\theta_{i}(0)=1 / 6 P_{i-1}+2 / 3 P_{i}+1 / 6 P_{i+1}
$$

Further,

$$
\begin{aligned}
& \theta_{1}^{\prime}(0)=-1 / 2 P_{0}+1 / 2 P_{2}=0 \\
& \theta_{i-1}^{\prime}(0)=-1 / 2 P_{i-2}+1 / 2 P_{i}=0
\end{aligned}
$$

Combined type (7) with type (8), we can get:

$$
\left[\begin{array}{cccccccc}
-3 & 0 & 3 & 0 & \mathrm{~L} & 0 & 0 & 0 \\
1 & 4 & 1 & 0 & 0 & \mathrm{~L} & 0 & 0 \\
0 & 1 & 4 & 1 & 0 & 0 & \mathrm{~L} & 0 \\
\mathrm{M} & \mathrm{M} & \mathrm{M} & \mathrm{M} & \mathrm{M} & \mathrm{M} & \mathrm{M} & \mathrm{M} \\
0 & 0 & \mathrm{~L} & 0 & 1 & 4 & 1 & 0 \\
0 & 0 & 0 & \mathrm{~L} & 0 & 1 & 4 & 1 \\
0 & 0 & 0 & \mathrm{~L} & 0 & -3 & 0 & 3
\end{array}\right]\left[\begin{array}{c}
P_{0} \\
P_{1} \\
P_{2} \\
\mathrm{M} \\
P_{i-1} \\
P_{i} \\
P_{i+1}
\end{array}\right]=\left[\begin{array}{c}
0 \\
M_{1} \\
M_{2} \\
\mathrm{M} \\
M_{i-1} \\
M_{i} \\
0
\end{array}\right]
$$

In type (9), $M_{1}, M_{2} \mathrm{~L} M_{i}$ is known quantity, so the only solution is the control points of the cubic-B spline trajectory.

Put control points into type (9) combined with the basic function we can get cubic-B spline curve:

$$
\theta_{i}(t)=1 / 6\left[\begin{array}{llll}
t^{3} & t^{2} & t & 1
\end{array}\right]\left[\begin{array}{cccc}
-1 & 3 & -3 & 1 \\
3 & -6 & 3 & 0 \\
-3 & 0 & 3 & 0 \\
1 & 4 & 1 & 0
\end{array}\right]\left[\begin{array}{c}
P_{i-1} \\
P_{i} \\
P_{i+1} \\
P_{i+2}
\end{array}\right]
$$

\section{Trajectory Plan}

One loading process of ammunition loading robot of a workflow can be separated into four parts: fetching, supplying, pulling and reset, of which the supplying stage need to pass the obstacle avoidance region. As shown in Figure 1, the end effector of ammunition loading robot starting point from $\mathrm{P}_{1}$ crossing obstacle avoidance area, finally reaching the $\mathrm{P}_{2}$, and then finish the loading action, in order to avoid the breechblock and seat ring, the end effecter must pass four key obstacle avoiding points $\mathrm{M}_{1}-\mathrm{M}_{4}$. 


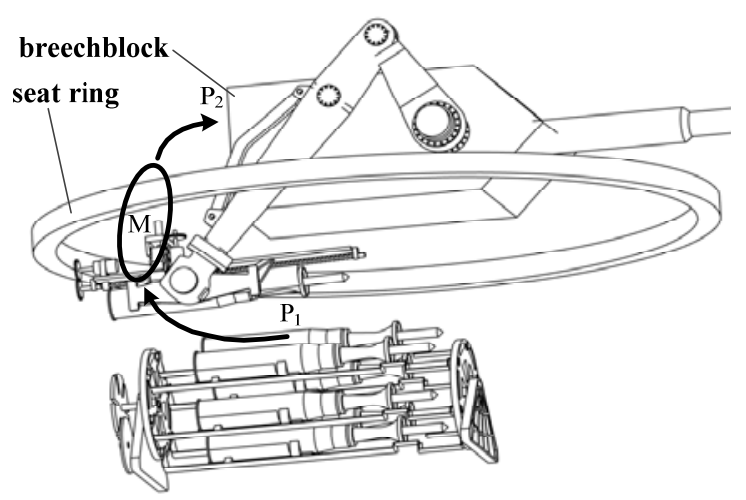

Fig.1. The supplying stage of ammunition loading robot

The joint variables corresponding to the starting point $\mathrm{P}_{1}$, the point $\mathrm{P}_{2}$ and the key obstacle avoidance point $\mathrm{M}_{1} \sim \mathrm{M}_{4}$ are solved by using the inverse kinematics of ammunition loading robot. See Table 1.

Table 1 The joint variables corresponding to the starting point $\mathrm{P}_{1}$, the point $\mathrm{P}_{2}$ and the key obstacle avoidance point $\mathrm{M}_{1} \sim \mathrm{M}_{4}$

\begin{tabular}{cccccccc}
\hline kepoints & $\mathrm{T}(\mathrm{s})$ & $\theta_{1}$ & $\theta_{2}$ & $\theta_{3}$ & $\theta_{4}$ & $\theta_{5}$ & $\theta_{6}$ \\
\hline $\mathrm{P}_{2}$ & 0 & 50 & 55.8 & -70.6 & 20 & 0 & 0 \\
$\mathrm{M}_{1}$ & 0.5 & 50 & 43.4 & -49.6 & 15 & 20 & 10 \\
$\mathrm{M}_{2}$ & 1 & 50 & 58.2 & -37.8 & 34.3 & 30 & 10 \\
$\mathrm{M}_{3}$ & 1.5 & 50 & 48.3 & -27.2 & 42.9 & 30 & 10 \\
$\mathrm{M}_{4}$ & 2 & 50 & 32.8 & -18.5 & 15.4 & 20 & 0 \\
$\mathrm{P}_{3}$ & 2.5 & 50 & 52.4 & -20.5 & 29.6 & 0 & 0 \\
\hline
\end{tabular}

Solving the control points of the cubic-B spline trajectory:

\begin{tabular}{|c|c|c|c|c|c|c|c|}
\hline $\mathrm{P}_{1}=[31.4287$ & 67.9856 & 31.4287 & 66.6995 & 50.9732 & 19.2077 & 68.9962 & 19.2077] \\
\hline$P_{2}=[-96.7498$ & -140.7751 & 96.7498 & -111.2258 & -76.9469 & -51.9866 & -74.5067 & $-51.9866]$ \\
\hline$P_{3}=[37.6000$ & 86.2000 & 37.6000 & 74.8000 & 90.4000 & 34.6000 & 10.6000 & $34.6000]$ \\
\hline
\end{tabular}

Trajectory planning is completed in joint space of ammunition loading robot based on cubic-B spline, and 2th, 3th and 4th joint trajectories are expressed as piecewise polynomial form:

$$
\begin{gathered}
\theta_{2}(t)= \begin{cases}193.2552 t^{3}-146.2284 t^{2}+55.8 & (0 \leq t \leq 0.5) \\
-163.7664(t-0.5)^{3}+143.6556(t-0.5)^{2}-1.287(t-0.5)+43.4 & (0.5 \leq t \leq 1) \\
46.6104(t-1)^{3}-101.9944(t-1)^{2}+19.5444(t-1)+58.2 & (1 \leq t \leq 1.5) \\
130.1240(t-1.5)^{3}-32.0784(t-1.5)^{2}-47.4918(t-1.5)+48.3 & (1.5 \leq t \leq 2) \\
-241.5080(t-2)^{3}+163.1080(t-2)^{2}+18.0230(t-2)+32.8 & (2 \leq t \leq 2.5)\end{cases} \\
\theta_{3}(t)= \begin{cases}-195.4024 t^{3}+176.1012 t^{2}-126.1 & (0 \leq t \leq 0.5) \\
143.008(t-0.5)^{3}-117.0028(t-0.5)^{2}+29.5494(t-0.5)-106.5 & (0.5 \leq t \leq 1) \\
-77.4312(t-1)^{3}+97.51(t-1)^{2}+19.8028(t-1)-103.1 & (1 \leq t \leq 1.5) \\
-50.8824(t-1.5)^{3}-18.6372(t-1.5)^{2}+59.2392(t-1.5)-78.5 & (1.5 \leq t \leq 2) \\
123.3608(t-2)^{3}-94.9608(t-2)^{2}+2.4402(t-2)-59.9 ; & (2 \leq t \leq 2.5)\end{cases} \\
\theta_{4}(t)= \begin{cases}244 t^{3}-194.4 t^{2}+70 & (0 \leq t \leq 0.5) \\
-143.2(t-0.5)^{3}+171.6(t-0.5)^{2}-11.4(t-0.5)+51.9 & (0.5 \leq t \leq 1) \\
-66.4(t-1)^{3}-43.2(t-1)^{2}+52.8(t-1)+71.2 & (1 \leq t \leq 1.5) \\
137.6(t-1.5)^{3}-142.8(t-1.5)^{2}-40.2(t-1.5)+78.5 & (1.5 \leq t \leq 2) \\
21.6(t-2)^{3}+63.6(t-2)^{2}-79.8(t-2)+39.9 & (2 \leq t \leq 2.5)\end{cases}
\end{gathered}
$$




\section{Simulations and Verification}

Based on the trajectories, the angle, angular velocity and angular acceleration of the 2th, 3 th and 4th joints of the ammunition loading robot are simulated, and the results are shown in Figure 2 to Figure 4.

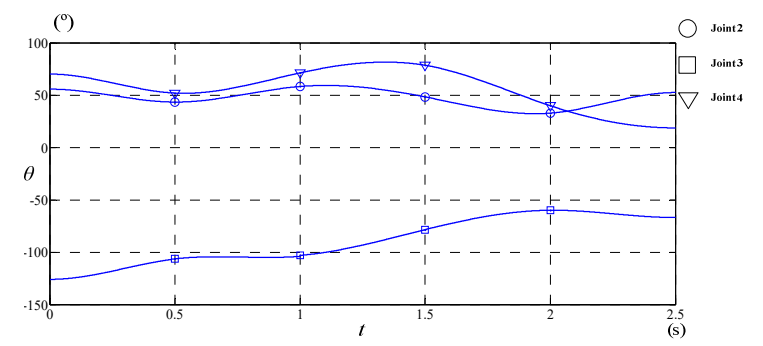

Fig.2. Joint angle-time relationship of trajectory

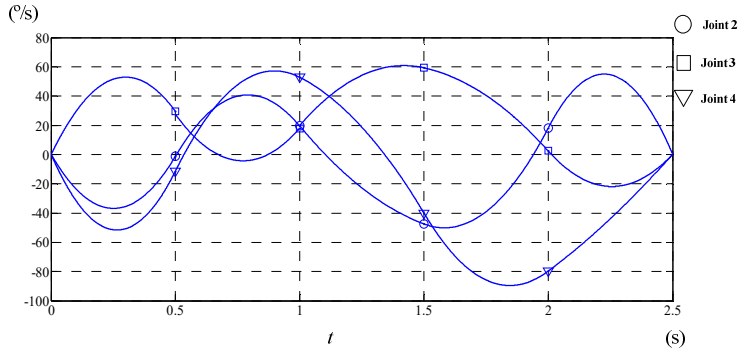

Fig.3. Joint angle velocity-time relationship of trajectory

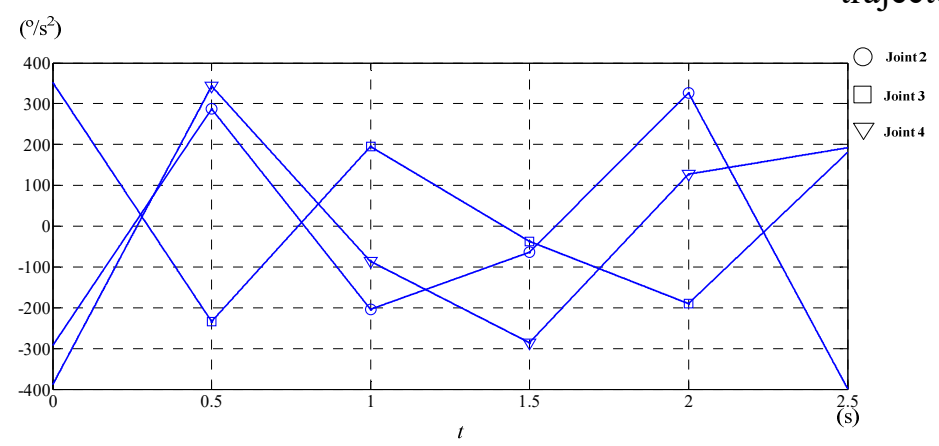

Fig.4. Joint angle acceleration-time relationship of trajectory

Figure 2 shows that the trajectories of ammunition loading robot in joint space using cubic-B spline are continuous, smooth, and pass all obstacle avoiding key points with no position mutation; Figure 3 shows that joint velocity curves are smooth, continuous, with no sudden change; Figure 4 shows that ammunition loading robot joint acceleration curve is continuous, with no sudden change. In summary, the joint space trajectory planning of ammunition loading robot based on the cubic-B spline is not only satisfy the kinematic requirements of ammunition loading robot, but also guarantee its good dynamic characteristics.

\section{Conclusions}

The paper put forward the obstacle avoidance trajectory planning method based on cubic-B, by which the trajectories planning in joint space of ammunition loading robot are finished. Simulate the 2 th, 3 th, 4th joints dynamics characters of ammunition loading robot by using dynamic simulation software, and the joint trajectory curves are continuous and smooth, angular velocity and angular acceleration curves are continuous with no sudden change, verifying that trajectories generating by cubic-B spline have good dynamic characteristic, making the ammunition loading robot in the working process no impacts, and guaranteeing ammunition loading robot complete loading task, also can prolong the service life of ammunition loading robot.

\section{Reference}

[1] Xu Da, Xia Xiang, Li Hua, Xiao Zi-qiang. Design and Implement of Simulation System for Ammunition Loading Robot Work Platforms [J]. Journal of Academy of Armored Force Engineering, 2012, 26(05): 55-58. 
[2] Dai Shi-jie, Zhang Yi, Wang Zhi-ping. Trajectory Planning of Welded Repair for Aero-engine Blades Based on NURBS Curve[J]. Transactions of the China Welding Institution. 2015, 36 (10): 23-24.

[3] J.J. Craig, Introduction to Robotics Mechanics and Control [M]. Pearson Education, State of New Jersey, 1998.

[4] Yi Xiao-li, Wang Ji-dai, Sun Jing. Mechanical design and trajectory planning of leg structure of bionic quadruped robot [J]. Journal of Mechanical Transmission, 2013, 37 (08): 34-38.

[5] Gao Dong-qiang, Yang Lei, Han Kun. The kinematics analysis and trajectory planning of SCARA manipulator [J]. Machinery Design \& Manufacture, 2015, 26(01): 269-262.

[6] Jia Qingxuan, Chen Gang, Sun Hanxu. Path Planning for Space Manipulator to Avoid Obstacle Based on A * Algorithm [J]. Journal of Mechanical Engineering, 2010, 46 (11): 109-115.

[7] V. Perdereau, C. Passi, M. Drouin. Real-time control of redundant robotic manipulators for mobile obstacle avoidance [J]. Robotics and Autonomous Systems, 2002, 41 (03): 41-59.

[8] Dai J S, Huang Z, Linkin H. Mobility of over constrained parallel mechanisms [J]. ASME Journal of Mechanical Design, 2006,128 (07): 200-299. 